\title{
Insight of transcriptional regulators reveals the tolerance mechanism of carpet-grass (Axonopus compressus) against drought
}

\author{
Mohsin Nawaz ${ }^{1}$, Liao Li ${ }^{1 *}$, Farrukh Azeem², Samina Shabbir ${ }^{3}$, Ali Zohaib ${ }^{4}$, Umair Ashraf $^{5}$, Hubiao Yang ${ }^{6}$ and \\ Zhiyong Wang ${ }^{1 *}$
}

\begin{abstract}
Background: Carpet grass [Axonopus compressus (L.)] is an important warm-season perennial grass around the world and is known for its adaptability to varied environmental conditions. However, Carpet grass lacks enough data in public data banks, which confined our comprehension of the mechanisms of environmental adaptations, gene discovery, and development of molecular markers. In current study, the DEGs (differentially expressed genes) in Axonopus compressus under drought stress (DS) were identified and compared with CK (control) by RNA-Seq.

Results: A total of 263,835 unigenes were identified in Axonopus compressus, and 201,303 (also added to the numbers of the remaining 2 databases) a sequence of unigenes significantly matched in at least one of the seven databases. A total of 153,697 (58.25\%) unigenes classified to 144 KEGG pathways, and 7444 unigenes were expressed differentially between DS and CK, of which 4249 were up-regulated and 3195 were down-regulated unigenes. Of the 50 significantly enriched GO terms, 18, 6, and 14 items were related to BP, CC, and MF respectively. Analysis of KEGG enrichment revealed 2569 DEGs involved in 143 different pathways, under drought stress. 2747 DEGs were up-regulated and 2502 DEGs were downregulated. Moreover, we identified 352 transcription factors (TFs) in Axonopus compressus, of which 270 were differentially expressed between CK and DS. The qRT-PCR validation experiment also supports the transcriptional response of Axonopus compressus against drought. Accuracy of transcriptome unigenes of Axonopus compressus was assessed with BLAST, which showed 3300 sequences of Axonopus compressus in the NCBI.
\end{abstract}

Conclusion: The 7444 unigenes were found to be between DS and CK treatments, which indicate the existence of a strong mechanism of drought tolerance in Axonopus compressus. The current findings provide the first framework for further investigations for the particular roles of these unigenes in Axonopus compressus in response to drought.

Keywords: Drought, Axonopus compressus, qRT-PCR, Enrichment analysis, IncRNAs, miRNAs, Transcriptomics

\footnotetext{
* Correspondence: liaoli@hainanu.edu.cn; wangzhiyong@hainanu.edu.cn

${ }^{1}$ Key Laboratory of Genetics and Germplasm Innovation of Tropical Special

Forest Trees and Ornamental Plants, Ministry of Education, College of

Forestry and College of Tropical Crops, Hainan University, Haikou 570228,

People's Republic of China

Full list of author information is available at the end of the article
}

C C The Author(s). 2021 Open Access This article is licensed under a Creative Commons Attribution 4.0 International License, which permits use, sharing, adaptation, distribution and reproduction in any medium or format, as long as you give appropriate credit to the original author(s) and the source, provide a link to the Creative Commons licence, and indicate if changes were made. The images or other third party material in this article are included in the article's Creative Commons licence, unless indicated otherwise in a credit line to the material. If material is not included in the article's Creative Commons licence and your intended use is not permitted by statutory regulation or exceeds the permitted use, you will need to obtain permission directly from the copyright holder. To view a copy of this licence, visit http://creativecommons.org/licenses/by/4.0/ The Creative Commons Public Domain Dedication waiver (http://creativecommons.org/publicdomain/zero/1.0/) applies to the data made available in this article, unless otherwise stated in a credit line to the data. 


\section{Highlights}

1. Axonopus compressus can stand severe drought stress by activating the potential defense mechanisms.

2. We investigated the differential transcriptome of drought-stressed and normal Axonopus compressus plants

3. New comers involved in the drought-response have been identified

4. The identified drought-responsive genes are not known for other stresses.

5. The identified genes also respond to stress in Arabidopsis thaliana in a different manner.

\section{Background}

Axonopus is a genus of the grass family (Paniceae; Poaceae; Triticeae). Approximately 100 species of Axonopus have already been identified [1], which are generally distributed along the shoreline of China, Africa, Alaska, central Asia, eastern Asia, North Sea, South America, and Oceania [2,3]. About more than 40\% (55.5 × 106 $\mathrm{km}^{2}$ ) of the Earth's surface is occupied by Grasslands world-wide, excluding Greenland and Antarctica [4].

The social and ecological susceptibility to climate change is a giant issue of modern era. The grassland gives an additional opinion about the vulnerability of the ecosystem on account of alterations in policies, socioeconomic elements, land use, and local climate [4]. Since the exposure to climatic hazards in various regions has become more apparent, the ecosystems are apt to have a greater degree of vulnerability. Globally, the number of socio-ecological attributes of the eco-systems have changed drastically due to lack of enough soil moisture [5].

Plant growth, nutrient balance, and photosynthetic dynamics have been influenced by drought [6]. Tolerant plant species with drought-responsive mechanisms at various levels including morphological, physiological, and molecular basis can be used to cope with water deficit. Given the important role of carpet-grass in ecosystem protection, scientists (from a macro point of view) are investigating how carpet-grass responds to global changes including drought, salinity, elevated temperature, and $\mathrm{CO}_{2}$ elevation $[7,8]$. However, little attention has been given to understand the genetic basis of its ecological diversifications, mainly because of the confined genomic resources in carpet-grass. Thus far, only limited ESTs and protein sequences from carpet-grass have been submitted in public online-databases [9]. The discovery of different genes is also lagging, and just a few genes happen to be cloned and functionally authenticated $[10,11]$.

High-throughput technologies of next-generation sequencing (NGS) like ABI/SOLiD, Roche/454, and Illumina/Solexa have facilitated the production of sizable genome resources at a comparatively low cost $[12,13]$. The next-generation sequencing has already been efficiently utilized to create large-scale transcriptome details in a number of plant species like rice [14], Arabidopsis $[15,16]$, wheat [17], barley [18], and maize [19].

Plants attempt to re-program their metabolic activities and growth while confronting water deficit conditions. It happens to be obvious that plants show distinct and extremely dynamic responses to limited water conditions [20]. These responses varied due to many factors including genotypes, experimental procedures, sampling technique, and time [20,21]. The molecular mechanisms in response to drought stress have been studied by many scientists to characterize the genes associated with water uptake [22], transporter channels [23, 24], and transcription factors [25]. All these attributes are regarded as the regulators of drought tolerance in plants, although a number of molecular elements and gene networks of drought tolerance mechanisms have been identified but still not fully elucidated [21].

\section{Methods}

\section{Plant materials and drought treatments}

Thirty days old Axonopus compressus L. plants with uniform growth were acquired from the germplasm resource library maintained at the Hainan University. The germplasm resource library is maintained by Key Laboratory of Genetics and Germplasm Innovation of Tropical Special Forest Trees and Ornamental Plants, Ministry of Education, College of Forestry, Hainan University, Haikou 570,228, P. R. China. Plants were grown in chambers with controlled conditions $\left(27^{\circ} \mathrm{C}, 16\right.$-h day length, and $60 \% \mathrm{RH})$. A total of 90 Axonopus compressus L. plants were primarily divided into two groups as CK (control group) and DS (drought experiment group). There were 5 cuttings in each pot and 20 cuttings in one replicate group. This experiment included 3 repeats named R1, R2, and R3. The drought treatments were induced by using the Polyethylene-glycol (PEG-8000). The PEG-8000 solution was replaced after $48 \mathrm{~h}$. At eight time points $(0 \mathrm{~h}, 6 \mathrm{~h}, 12 \mathrm{~h}, 18 \mathrm{~h}, 24 \mathrm{~h}, 36 \mathrm{~h}, 48 \mathrm{~h}$, and $72 \mathrm{~h}$ after induction of drought treatment) the functional leaves (3rd to 5th mature leaf) were chosen randomly from the plants of CK and DS for physiological attributes. For transcriptome sequencing, samples were instantly frozen in liquid nitrogen, stored at $-80^{\circ} \mathrm{C}$, and finally sent to Metware Biotechnology Co., Ltd., Gaoxin Road, East Lake High-tech Zone, Wuhan, China.

\section{Measurement of physiological traits}

The physiological traits were recorded in different time intervals of $0 \mathrm{~h}, 6 \mathrm{~h}, 12 \mathrm{~h}, 18 \mathrm{~h}, 24 \mathrm{~h}, 36 \mathrm{~h}, 48 \mathrm{~h}$, and $72 \mathrm{~h}$ after induction of drought. The leaf water potential (LWP) was measured by Scholander chamber (SF-PRES- 
70, Solfranc Tecnologías SL, Vila-Seca, Spain). Electrolyte leakage (EL) was measured by estimating the electrical conductivity [26]. In the solution, the electrolyte leakage (S1) was measured using a conducti-meter after $22 \mathrm{~h}$ of floating at room temperature (Mettler-Toledo Instruments Co., Ltd., Shanghai, China). Total conductivity (S2) was collected after the flasks were placed in a boiling water bath for $30 \mathrm{~min}$. Relative water content (RWC) was also estimated, as described in Liu et al. [27]. For each plant, three independent replicates were collected for LWP, EL, and RWC. All measurements were recorded at three biological replicates.

\section{Extraction of RNA of Axonopus compressus, library preparation}

Total RNA of Axonopus compressus was extracted by employing the Trizol method (Invitrogen, Carlsbad, CA, USA). The RNA integrity, purity and concentration were accessed by the Nanodrop spectrophotometer (Qubit 2.0, Agilent 2100). The sequencing libraries of Axonopus compressus were created by NEBNext ${ }^{\circ} \mathrm{Ultra}^{\mathrm{Tm}} \mathrm{RNA} \mathrm{Li}$ brary Prep Kit for Illumina (New England Biolabs, Ipswich, MA, USA), as suggested by the manufacturer. The first strand of cDNA was synthesized by random hexamer primer, afterwards, the dNTPs, buffer, DNA polymerase I, and RNase $\mathrm{H}$ were used to generate cDNA's second strand. Finally, the AMPure XP beads were used to purify cDNAs and afterward the end-repair and single nucleotide A (adenine) addition, the cDNA libraries (qualified) were developed by PCR technique. The Qubit 2.0 was used for primary quantitation, followed by Agilent 2100, which was employed to identify libraries insert size. Thereafter, the q-PCR technique had been used for accurate quantification of the effective strength of the libraries (effective library concentration $>2 \mathrm{nM}$ ) to guarantee the library quality. After passing through a series of screening steps, the high-throughput sequencing was executed by Illumina Hiseq Xten. The protein-coding region accuracy and completeness assessment in Axonopus compressus was performed by using BUSCO analysis (BUSCO_v2/v3) [28].

\section{Validation of RNA-seq data by qRT-PCR}

The RNA was extracted from the CK and DS groups and used to develop the cDNA library. First-strand synthesis was carried out via MonScript (Monad) according to the suggested protocol by the manufacturer. Biosystems-7500 (Thermofisher Scientific) was used to conduct the qRTPCR and the primer pairs are listed in Additional file 1; Table S1. The gene expression was quantified through CT-method, and the Actin1 was used as a reference for normalization.

\section{Results \\ Effect of drought stress on physiological indicators in Axonopus compressus}

In Fig. 1, it was revealed that the rate of $\mathrm{H}_{2} \mathrm{O}_{2}$ (Fig. 1a) production and MDA concentration (Fig. 1b) increased in drought treated plants. The MDA values and $\mathrm{H}_{2} \mathrm{O}_{2}$ production rate increase with the prolonged drought stress treatment, whereas MDA values and the production rate of $\mathrm{H}_{2} \mathrm{O}_{2}$ at $36 \mathrm{~h}$ after drought induction were significantly increased $(p<0.01)$. Similarly, the leaf water potential and relative water content decrease significantly under drought, contrarily, the electrolyte leakage increased over the drought period in Axonopus compressus. As in the case of MDA and $\mathrm{H}_{2} \mathrm{O}_{2}$, the values of EL were also increased as the time of drought stress increase. Whilst, the values of leaf water potential and relative water content decrease with the prolonged drought stress (Fig. 1c).

RNA-Seq of Axonopus compressus and de novo assembly RNA-Seq of the six libraries of cDNA (DS and CK with three replicates each) resulted in 352.84 million raw reads, of which approximately 347.73 million clean reads de-novo compiled into contigs utilizing Trinity software, tend to range from 54.90 million to 62.25 million reads for each library and approximately $97 \%$ of reads with a quality score of Q20 (99\% accuracy) (Additional file 1; Table S2). The contigs were assembled into 278,042 unigenes with an average length of $1066 \mathrm{bp}$ and an N50 length of $1430 \mathrm{bp}$. All unigenes were over $200 \mathrm{bp}$ long, of which $11.77 \%$ (32,718 unigenes) were $2000 \mathrm{bp}$ long. We evaluated the assembled data of transcriptome by employing Benchmarking Universal Single-Copy Orthologs (BUSCO) package. The number of total BUSCOs searched was 1440. The number of complete BUSCOs in the combined transcriptome was $1080(75.00 \%)$, and only 198 core genes (13.75\%) are fragmented in Axonopus compressus (Additional file 2; Fig. S1).

\section{Functional annotation and classification of unigenes}

The assembly of unigenes from Axonopus compressus was annotated by BLASTX (E-value $<10^{-5}$ ) in different databases. A total of 135,716 (48.81\%), 188,334 (67.64\%), 120,455 (43.32\%), 189,422 (68.13\%), 100,588 (36.18\%), 153,958 (55.37\%) and 128,626 (46.26\%) unigenes would have significant levels $\left(\mathrm{E}\right.$-value $<10^{-5}$ ) against KEGG, NR, Swissprot, Trembl, KOG, GO, and Pfam, respectively (Fig. 2a). Out of 278,042 unique of high-quality sequences, 191,893 (69.03\%) unigenes complemented a sequence significantly in at least one of the seven public databases, The five key online databases (GO, KEGG, KOG, NR, and Trembl) were picked out of seven databases to draw a Venn diagram (Fig. 2b), the unigenes with significant values $\left(\mathrm{E}\right.$-value10 $\left.{ }^{-5}\right)$ are also noticed at 


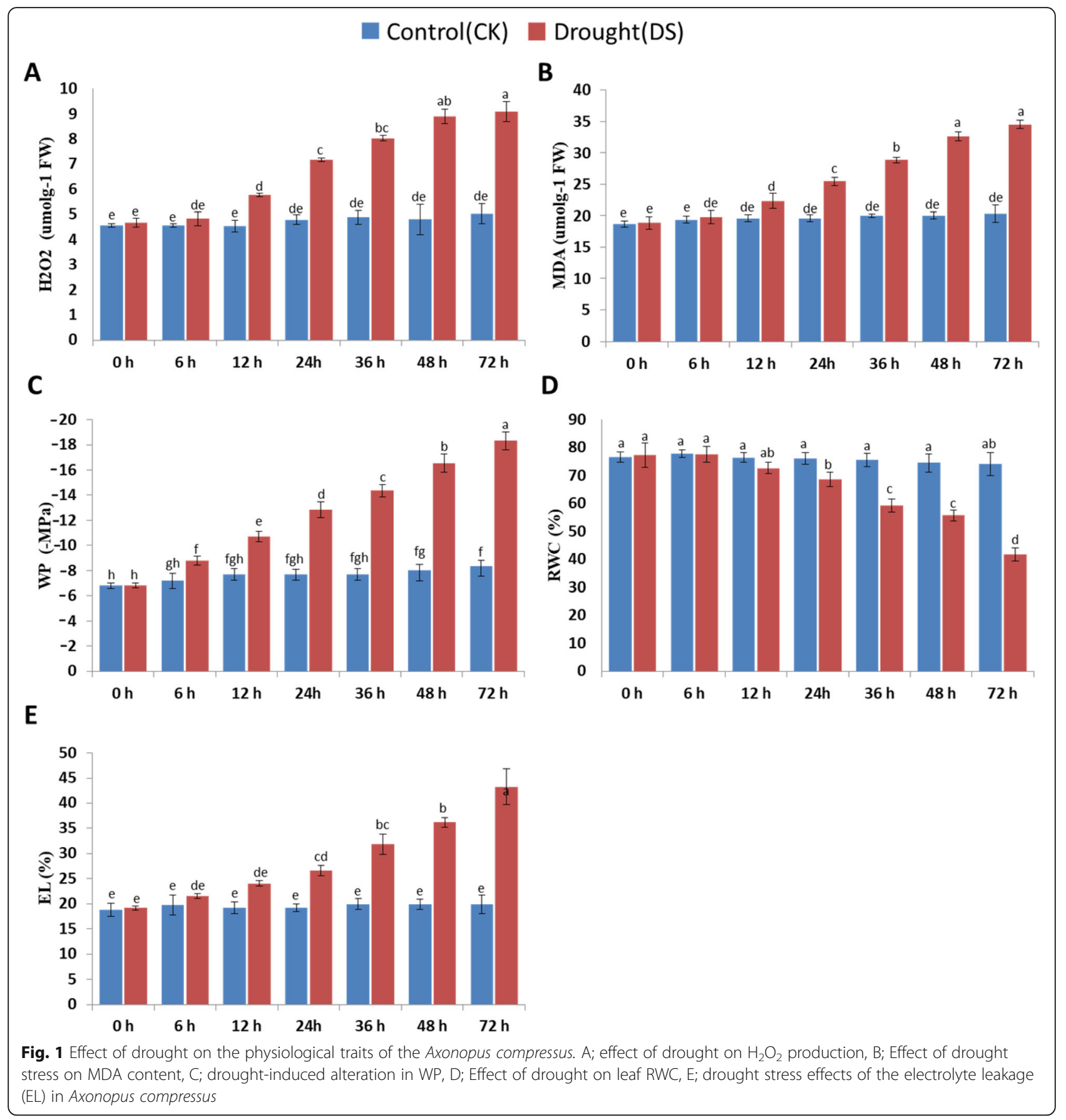

each junction of the Venn diagram, in which 94 unigenes corresponds to five databases.

The alignment of sequence homology revealed that 39 , $439(20.94 \%)$ sequences that were found in resemblance with Setaria italica; 35,283 (18.73\%) sequences had significant hits for Zea mays, followed by Sorghum bicolor (35,034, 18.6\%), Penicum hallii (23,894, 17.47\%), Dichanthelium oligosanthes $(18,896,10.03 \%)$ and Oryza sativa japonica group (6551, 3.48\%). Brachypodium dischyon which included 1675 (0.89) and $9.86 \%$ of the sequences (18562) were homologous to other species (Fig. 2c). The expression of genes has biological variability between different individuals, and there are differences in the degree of expression between genes. The reads per kilobase of exon model per million of aligned reads (FPKM) values were calculated as normalized expression estimates for each gene model in each sample. To evaluate the major spectral variance between CK and DS treatment samples, we performed PCA (Fig. 2d). Results showed a distinct separation of CK to DS sample 


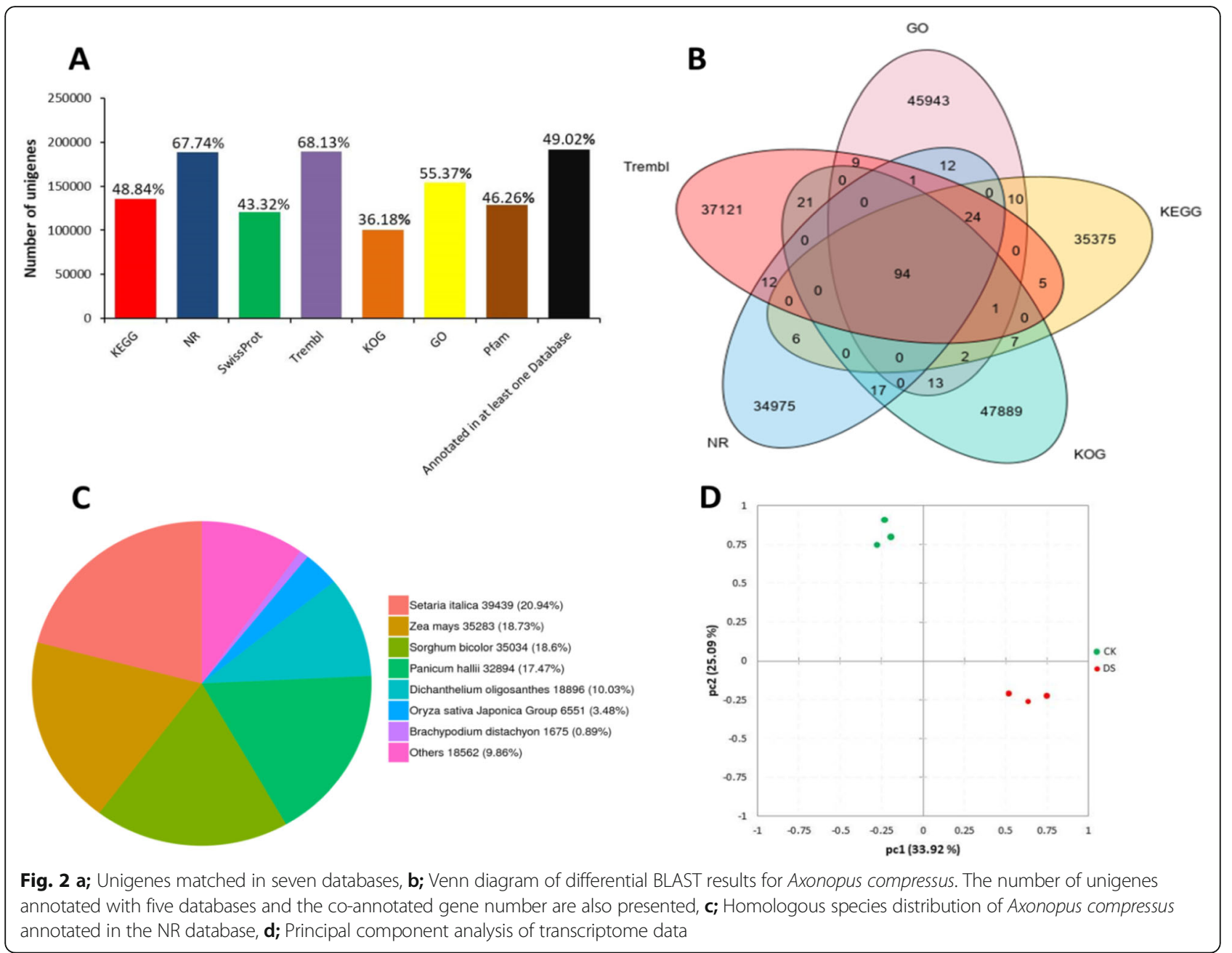

treatments, which reveals that both groups of the treatment samples exhibit different spectral positions on the PCA chart (Fig. 2d).

To demonstrate the accuracy of the unigenes prediction, the nucleotide sequences from the transcriptome of Axonopus compressus were used as a query in a BLAST search with a threshold E-value $<10^{-5}$. The results showed 3300 sequences of Axonopus compressus in the NCBI database. This analysis renders an assessment for the consistency of unigenes sequences in the current dataset. Based on the high-score BLASTx matches in the GO proteins database, The BLASTx high-score Predicated matches in the computer database of Go proteins were confirmed, and a total of 153,958 unigenes were categorized with Blast2GO (Evalue $<10^{-5}$ ) and were designated at least once in GO. As shown in Fig. 3, the unigenes referred to three main categories of GO and 59 subcategories, namely biological processes (BP), with 28 main sub-classes $(405,025$ unigenes); cellular compartments (CC), with 18 main subclasses (460,094 unigenes); and molecular functions (MF), with 13 main sub-classes (194,989 unigenes).
Cellular processes $(21.43 \%)$ were the biggest subgroups in the category of biological processes, metabolic process (19.70\%), biological regulation (9.24\%), response to stimulus $(9.22 \%)$, and regulation of biological process $(8.36 \%)$. The largest subgroups in the cellular component category were cell (22.53\%) followed by cell part (22.47\%), organelle (17.22\%), and membranes $(11.72 \%)$ respectively. Similarly, the main subgroups in the molecular function category were binding and catalytic activity, which contribute 77.78 and $69.28 \%$ respectively, and 115,199 unigenes associated with molecular function. Within the Axonopus compressus unigenes, 112,492 (42.64\%) were classified (E-value $<10-5)$ into twenty-six KOG clusters (Fig. $3 \mathrm{~b})$. The biggest groups which includes; 1) general function prediction, only (21,955 genes, $19.52 \%)$; 2) posttranslational modification, protein turnover, and chaperones (11,636 genes, $10.34 \%)$; 3 ) signal transduction mechanisms (11,302 genes, 10.05\%); 4) Function unknown (5785 genes, 5.14\%); and 5) carbohydrate transport and metabolism (5616 genes, 4.99\%). 


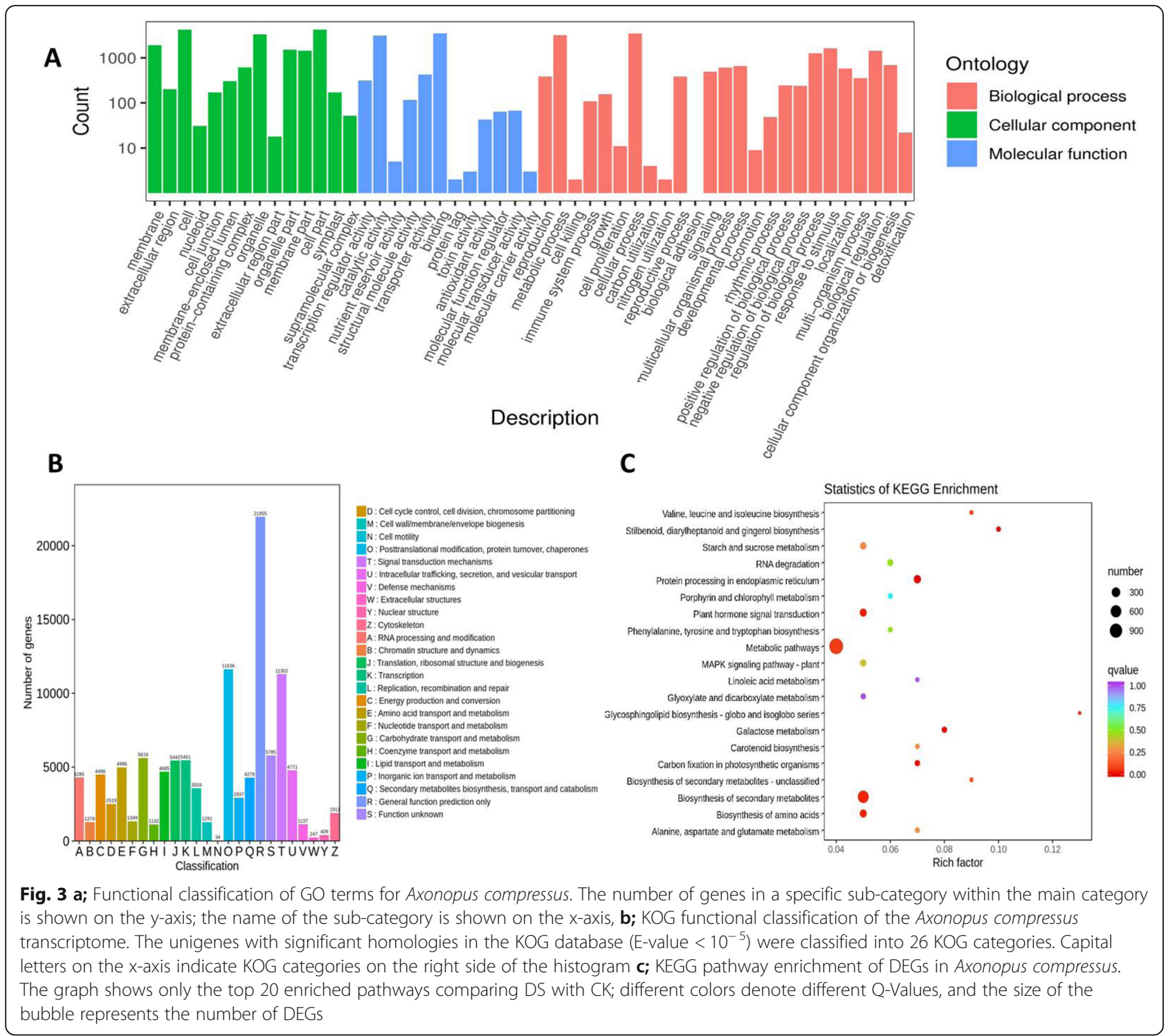

\section{Metabolic pathway analysis of Axonopus compressus by KEGG}

A total of 135,717 (51.44\%) of the 263,835 Axonopus compressus unigenes possessed significant correspondence in KO. All these unigenes have been restricted to 103 KEGG pathways in 5 major categories (Fig. 3b). The pathways of KEGG (which includes 4303 unigenes) were the members of a major group, metabolism (D), 921 associated to genetic information processing (C), 184 related to cellular processes (A), 347 involved to environmental information (B), and 151 related to category (E) of organism systems (Additional file 2; Fig. S2).

\section{CDS prediction in Axonopus compressus}

The BLASTx protein database (NR and SwissProt database) identified 278,042 unigenous CDSs, of which 23, 652 unigenes were larger than $500 \mathrm{bp}, 12,520$ unigenes were larger than $1000 \mathrm{bp}$, and 32,718 unigenes were larger than $2000 \mathrm{bp}$. In addition, 43,713 unigenes were not linked to the NR and SwissProt database systems. The Estscan (Version; 3.0.3) software was used to interpret their ORF, frequency distribution, length, and related amino acid sequences of the unigene CDSs.

\section{Differentially expressed genes (DEGs) analysis of Axonopus compressus}

Amongst the differentially expressed unigenes, the expression of 7444 differs substantially between samples treated with drought-stress (DS) and control (CK) samples. Under drought treatment, 4249 numbers were upregulated and 3195 numbers were down-regulated $(p<0.05)$ (Fig. 4a). The expression profiles of DEGs were also presented through cluster analysis that showed 


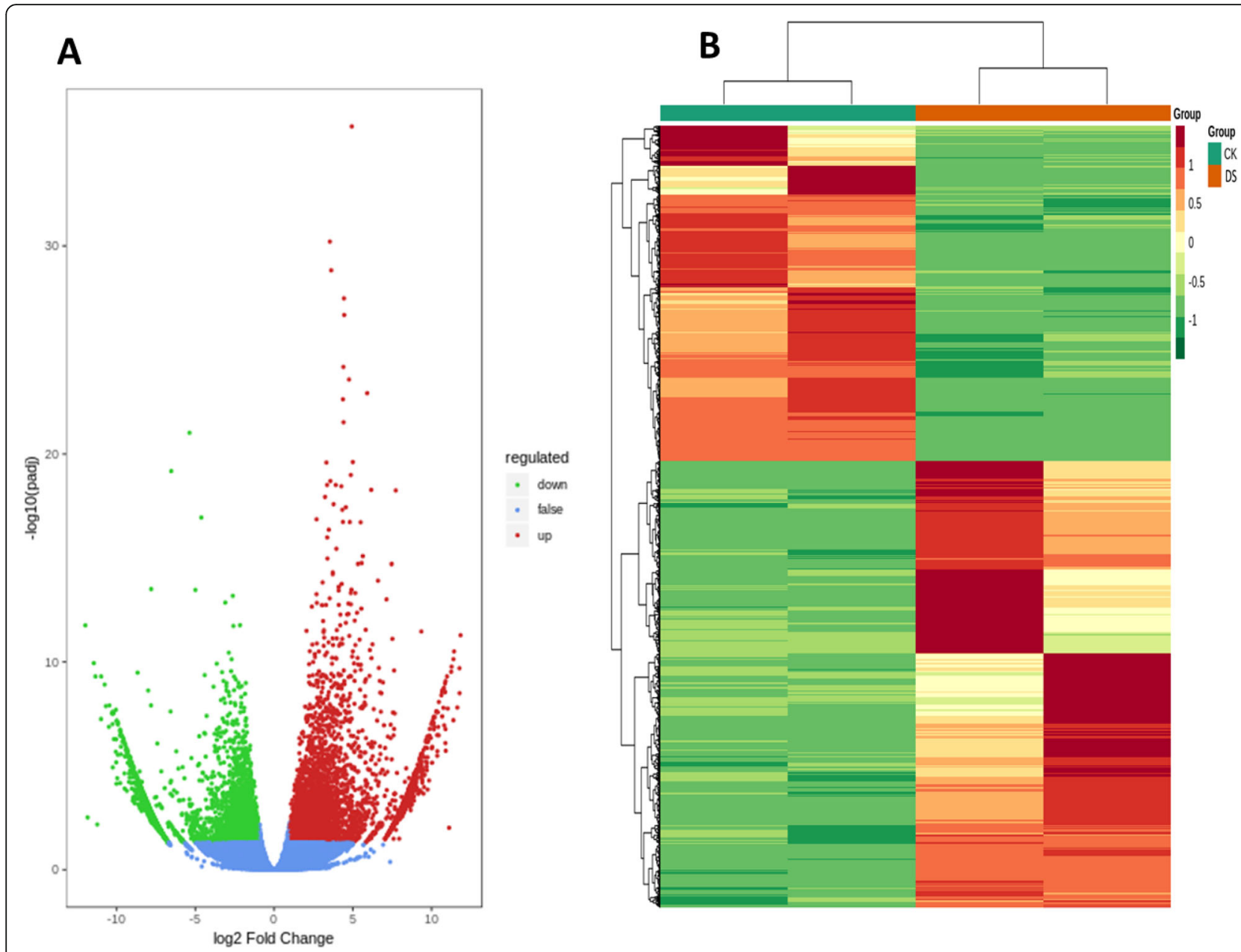

Fig. 4 a Volcano plot of differentially expressed unigenes of Axonopus compressus. The numbers of up and down-regulated unigenes in CK and DS are shown. Red indicates up-regulated genes, green represents down-regulated unigenes, and blue represents no significant difference in gene expression, $\mathbf{b}$; cluster analysis of differentially expressed unigenes of Axonopus compressus in responses to CK and DS treatments

significantly different responses of CK and DS treatments in the Axonopus compressus (Fig. 4b).

\section{GO pathway enrichment analysis}

By employing the Gene Ontology (GO) and the DEG enrichment analysis in Axonopus compressus, 23,766 DEGs were categorized into three GO groups and 4592 numbers associated the DS with CK (number of DEGs annotated in more than one term), out of which 2766 items that were associated to BP, 477 were linked with $\mathrm{CC}$ and 1349 were related to MF.

The key 50 DEGs dramatically enriched in three GO categories are presented in Additional file 2; Fig. S4. In the top 50 significantly enriched identities (Corrected $P$ Value< 0.05), 18 were found to be linked with BP [carotenoid biosynthetic process GO:0016117, carotenoid metabolic process GO:0016116, cellular amine metabolic process GO:0044106, cellular biogenic amine metabolic process GO:0006576, cellular response to heat GO:
0034605, cellular transition metal ion homeostasis GO: 0046916, glutamine family amino acid biosynthetic process GO:0009084, Group II intron splicing GO: 0000373, heat acclimation GO:0010286, oligosaccharide catabolic process GO:0009313, raffinose catabolic process GO:0034484, raffinose metabolic process GO: 0033530, regulation of seed germination GO:0010029, regulation of seedling development GO:1900140, response to high light intensity GO:0009644, response to hydrogen peroxide GO:0042542, tetraterpenoid biosynthetic process GO:0016109, tetraterpenoid metabolic process GO:0016108], and 6 items were related to CC [chloroplast nucleoid GO:0042644, DNA packaging complex GO:0044815, Nucleoid GO:0009295, Nucleosome GO:0000786, plastid nucleoid GO:0042646, protein-DNA complex GO:0032993], and 14 items were related to MF [4-coumarate-CoA ligase activity GO: 0016207, alpha-galactosidase activity GO:0004557, arogenate dehydratase activity GO:0047769, carbon- 
nitrogen ligase activity, with glutamine as amido- $\mathrm{N}$ donor GO:0016884, ferric iron binding GO:0008199, ferroxidase activity GO:0004322, galactosidase activity GO: 0015925, oxidoreductase activity, acting on single donors with incorporation of molecular oxygen GO:0016701, oxidoreductase response, acting on single donors with molecular oxygen incorporation, incorporation of two atoms of oxygen GO:0016702, oxidoreductase response, oxidizing metal ions, oxygen as acceptor GO:0016724, raffinose alpha-galactosidase activity GO:0052692, water channel activity GO:0015250, water transmembrane transporter activity GO:0005372] (Additional file 3).

\section{Axonopus compressus KEGG pathway enrichment analysis}

The KEGG pathway enrichment analysis for DEGs revealed that 2569 DEGs participated in 143 various types of pathways in Axonopus compressus. By comparing DS with CK, 2747 DEGs were found to be up-regulated and 2502 DEGs have been identified as down-regulated in deficit water. The twenty key pathways which were found significantly enriched by comparing DS with CK are displayed in (Fig. 3c). The pathways includes Valine, leucine and isoleucine biosynthesis (215 unigenes), Stilbenoid, diarylheptanoid and gingerol biosynthesis (330 unigenes), Starch and sucrose metabolism (1642 unigenes), RNA degradation (1536 unigenes), Protein processing in endoplasmic reticulum (1628 unigenes), Porphyrin and chlorophyll metabolism (559 unigenes), Plant hormone signal transduction (1672 unigenes), Phenylalanine, tyrosine and tryptophan biosynthesis (601 unigenes), Metabolic pathways (1659 unigenes), MAPK signaling pathway plant (1661 unigenes), Linoleic acid metabolism (323 unigenes), Glyoxylate and dicarboxylate metabolism (1050 unigenes), Glycosphingolipid biosynthesis - globo and isoglobo series (103 unigenes), Galactose metabolism (735 unigenes), Carotenoid biosynthesis (506 unigenes), Carbon fixation in photosynthetic organisms (820 unigenes), Biosynthesis of secondary metabolites - unclassified (223 unigenes), Biosynthesis of secondary metabolites (1661 unigenes), Biosynthesis of amino acids (1655 unigenes), Alanine, aspartate and glutamate metabolism (639 unigenes) (Additional file 2; Fig. S3).

\section{Drought stress associated differentially expressed transcription factors (TFs)}

The transcription factors play a crucial role in the growth and development of plants and therefore can stimulate and/or suppress transcriptional gene expression to sustain normal physiological functions in deficit water stress. In the current study, $352 \mathrm{TFs}$ were observed in Axonopus compressus based on iTAK, including 270 transcription factors and 81 transcription regulatory factors that were associated with 32 and 16 families, respectively (Additional file 1; Table S3, S4).
The differential analysis revealed that 270 transcription factors were different as shown in the comparison between DS and CK, 216 transcription factors have been noted to be up-regulated while 54 transcription factors were found down-regulated.

\section{qRT-PCR validation of transcripts in Axonopus compressus under drought}

To check the validity of RNA-seq results, the expression of six transcripts for the three biological replicates was analyzed by qRT-PCR (Fig. 5). All the genes were identified as significantly different expression values (DEGs). The findings of qRT-PCR revealed that the pattern of expression of these genes was identical with the findings in the RNA-seq analysis. To validate the results, the six drought-responsive genes (NAC, MAP Kinase1, MYB2, PIP1, WRKY1, ABI5) were verified using qRT-PCR. The different genes were amplified to compare the gene expression and RNA-seq results, and the findings of this study revealed that the expression of drought-responsive genes supporting the results of the RNA-seq.

\section{Discussion}

Plants have a sessile lifestyle, and usually confront adverse environmental circumstances such as drought, cold, heat, salt, and floods. Deficit water is the most notable environmental determinant influencing crop production around the globe [29]. To improve the potential tolerance mechanism against deficit water stress and also to develop drought-resistance in plants, many experts have attempted to elucidate the mechanisms of stress signaling in plants conferring several perspectives [30, 31]. Plant defense mechanisms for abiotic stress are already being studied, but the response to drought has always been a complex event with several critical indicators to be examined.

Drought stress significantly increases ROS formation and imposes oxidative stress on the plant [26, 32]. Since lipid peroxidation is one of the earliest indicators of oxidative damage, MDA was estimated as an index for the production of drought-induced ROS [32]. A significant rise in MDA values and $\mathrm{H}_{2} \mathrm{O}_{2}$ concentration were noticed in Axonopus compressus leaves in drought conditions (Fig. 1b). Electrolyte leakage increased significantly with a decline in water potential (Fig. 1e), which proposed that plant systems had been damaged by drought, as studied previously [33]. Drought might have had an impact on the physical membranous structure of the lipid bilayer by inducing phase destruction [34].

To sustain under severe environmental conditions, plants have developed an obscure regularity mechanism through a series of evolutionary advancements at varied levels to respond to external signals and prompt transduction of stress signals, directing to a set of responses 


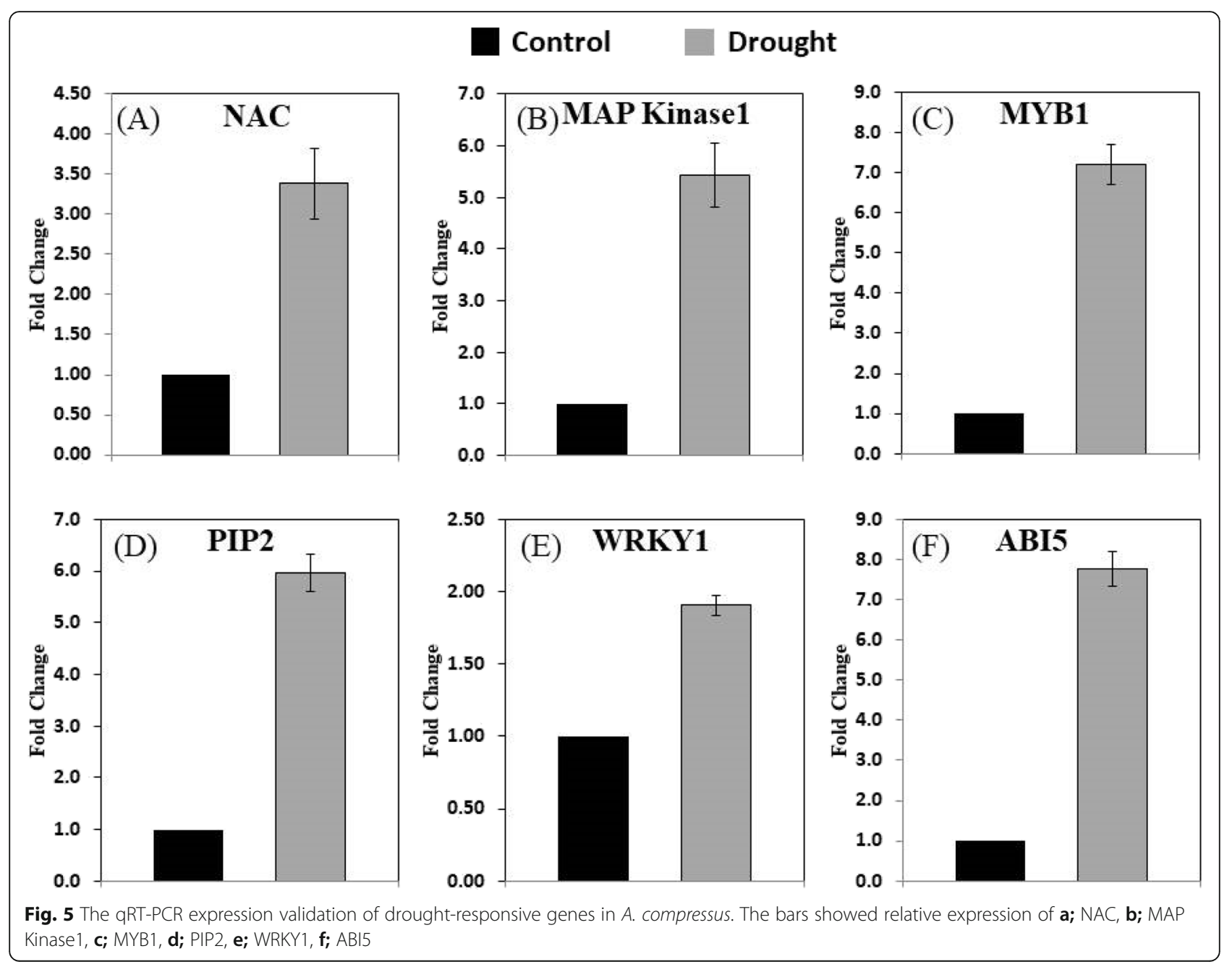

at different levels, i.e., physio- morphological, biochemical, and omic levels [35, 36]. As a strong droughtresponsive plant, Axonopus compressus developed a series of morphological features to acclimate the deficit water conditions. Drought stress can drive the plant to change the metabolic status of cutin and wax, which is ultimately accomplished through the associated geneexpression regulation [37] In current study, the unigene responsible for the wax biosynthesis (Cluster-37, 496.124448) was enriched under DS in comparison with CK and also up-regulated at the transcript level. As a distinctive morphological feature of protection against drought stress, cutin and wax play a critical role during water deficit conditions by restricting loss of water by means of leaf epidermis (non-stomatal transpiration) [38, 39], thereby enhancing water use capability in plants. In our study, the transcription factors that belong to the family AP2/ERF-ERF, MYB-related, zinc-finger, NF-X1 were found to be up-regulated. These transcription factors were known to regulate the biosynthesis of plant cutin and wax [40].
In water deficit conditions, plants receive stress signals first, and thereafter, several pathways in a sequence are triggered by phytohormones [41]. Subsequently, plant hormones play an important role in responding to scarce water conditions, and amongst the phytohormones, $\mathrm{ABA}$ is known to be a major homeostatic controller of abiotic stresses. In water deficit conditions, plants increase ABA content and close stomata to avoid the excessive water loss by transpiration across stomata [42], such a high level of water loss would sometimes continue to occur even after transient recovery of leaf water status [43].

In current study, we observed 8 unigenes, which encode NCED4 (9-cis-epoxycarotenoid dioxygenase) (Cluster-37,496.127826), a crucial enzyme accountable for ABA biosynthesis (GO:0009688). Amongst 8 unigenes, 4 were up-regulated and exhibited substantially different expression levels between DS and CK. Such comportment in response to drought stress indicates that Axonopus compressus plants had perceived the signals to produce $\mathrm{ABA}$ and to translocate it to the above-ground parts. The transportation mechanism in the stem comes 
into action and triggers short-term responses such as stomatal closure [41, 44], which potentially help Axonopus compressus to avert dehydration and improve water holding capacity under drought stress. Accordingly, the understanding of stress signaling and subsequent molecular mechanisms in Axonopus compressus need to be elucidated in succeeding experiments to reveal the drought-resistance mechanism.

When plants respond to drought, the rise in amounts of ABA leads to binding with PYR/PYL, which perform a significant role in quantitative regulation of stomatal movements and transcriptional response to $\mathrm{ABA}[45,46]$. The PYR/PYL reconstructs the PYR/PYL protein conformations, and this alteration enables the PYR/PYL to interplay with $2 \mathrm{C}$ protein phosphatase (PP2C), negative regulator type, to construct a substitute complex (ABA-PYR/PYLPP2C). The ABA-PYR/PYL-PP2C- complex may impede PP2C activity and trigger SNF1-related protein kinase 2 (SnRK2s), a positive regulator. While the PP2C also inhibits the activity of SnRK2s [47]. Such mechanism stimulates the stress-responsive genes to down-regulate its expression and supports the plants to acclimate water deficit conditions [48]. 715 PP2C were found in our experiments, from which 82 genes were remarkably downregulated, and 17 SnRK2 were also identified. This symbolizes that under water deficit, the low expression of PP2C in Axonopus compressus rescue the suppression of SnRK, and its expression promotes the closing of stomata. The SnRK expression also triggers the stress-related genes towards downstream and support Axonopus compressus keeps normal growth and persistence in drought.

In plants, the stress responding genes can easily be divided into two groups i.e., functional and regulatory [49]: The functional proteins are related to small molecular osmolytes (i.e., proline, betaine, and soluble sugar), enzyme protectants (POD, SOD and CAT), late embryogenesis abundant (LEA), and aquaporins. The regulatory proteins include TFs, phosphatases, protein kinases, and phospholipid metabolic enzymes that can regulate the stress-related expression of genes under abiotic stresses. It indicates that during water deficit conditions, the lowest expression of PP2C in Axonopus compressus would rescue the SnRK suppression. The PP2C expression would induce closing of stomata and trigger the downstream drought stress-related genes and assist the Axonopus compressus to sustain unhampered growth under drought. These proteins will prevent the plant cells from damaging encounters of stress by managing turgor pressure, including oxygen-free radicals scavenging and the structural intracellular biomacromolecules protection $[50,51]$.

It has been confirmed that members of the TF families i.e., MYB, AP2, DREB, bHLH, PLATZ, bZIP, C2H2, NAC WRKY, and $\mathrm{HB}$ are involved in plant stress- response mechanisms [52]. In our research, we found 270 DEGs in Axonopus compressus TFs, among them, 216 were up-regulated and 54 were down-regulated. It is obvious from the results that large numbers of TF expressions differed significantly under water deficit.

In the DEGs HARBI1, EEF1A, SUMO, ATXR3, SEY1, NLR, COMM, DHAR3, GUF1, SFH5, SHOC2, MAPK's, FYVE, PIP, LSM14, PERQ, BRCA1, TIM22, STY46-like, SPN1, NEDD8, DDX21, Meis2, RAD50, MAG2, MON1, and $A K T 2$ are all down-regulated. The RXW8, DHAR2like, EFR3, MOCS2A, GDSL, ANP1-like, TBC1, ERCC-6, ZDHHC2, EXO84B, BAHD, SHOC2, XBAT32, RNF38, SPIRAL1, NDRG1, HESO1, NBR1, STY46, TNNI3K, PP2C26, HVA22 and DAD1 are all up-regulated. The TF families i.e., GATA, ATF/CREB, ABI, bZIP, WRKY, EREBP, AP2-like, PTI6, TRAF, ARF1, ATF/CREB, ABAresponsive, RING, MYB, NAC, TUB, HSF, GRAS, C2H2, bZIP, SET, AP2/ERF-ERF, AUX/IAA, SNF2, and PTI6 some of them were up-regulated and some of them were down-regulated. A schematic diagram of potential defense mechanism and drought tolerance involving different pathways in Axonopus compressus is shown in Fig. 6. Several unigenes (belonging to a similar family) were reported to have distinct expression models under water deficit conditions. It might symbolize various characteristic functions in response to drought $[47,53]$. A particular transcription factor can be associated with one and/or more than one category and even other groups [54]. It reveals the complexity by which transcriptional categories of genes control the drought responses of Axonopus compressus. The current study provides helpful data for additional functional characterization of the TFs to develop tolerance in this plant.

During signal transduction in plants under stress conditions, phosphorylation and de-phosphorylation are very important mechanisms for post-translational protein modifications [55]. Phosphorylation and dephosphorylation can trigger a number of proteins and enzymes with regulatory functions to control a wide range of cellular mechanisms or signaling process. It has been established that protein phosphorylation plays a significant role in drought response to halt or initiate the enzyme activity, for adjusting the concentration of resultant proteins or intracellular enzyme activity [55]. It was reported that Arabidopsis has numrous kinases [56]. From which the CDPK (calcium-dependent protein kinase) and MAPK (Mitogen-activated protein kinase) are considered critical signaling mechanisms in plants under stress. The stress signals were transduced into cellular processes by MAPK, succeeding phosphorylation processes of distinct downstream proteins for turning on and/or off their activities [56, 57]. In our study, 11,089 protein kinases were observed, of which 344 were DEGs (182 up-regulated and 162 down-regulated). There were 


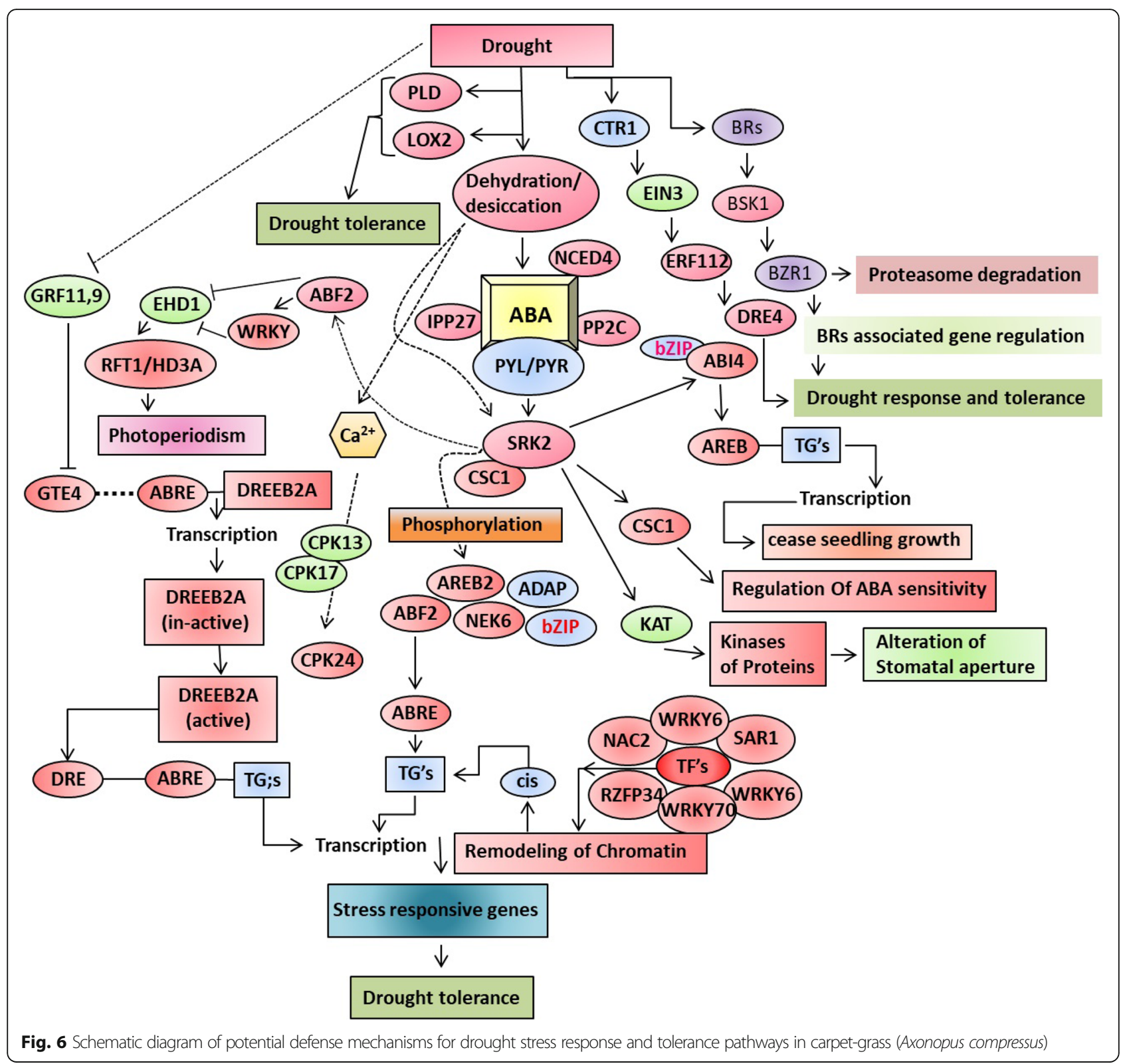

16 DEGs encoding MAPKs (9 up regulated and 7 down regulated) and 8 DEGs for CDPKs (2 up regulated and 6 down regulated). It suggests that the MAPKs and CDPKs pathways were engaged in the signaling of drought stress response of Axonopus compressus. When plants face water deficit conditions, osmo-tension would provoke ROS built-up in cells. Plants evolved the defense mechanism to extinguish the ROS and alleviate cellular damage. This defense mechanism comprises of SOD, POD, and CAT. The current study indentified DEGs related to 5 SODs, 3 CATs and 25 PODs. In addition, proteins of small-molecules such as proline, betaine, and LEA (late embryogenesis abundant) likewise play significant roles in restraining the cells from the damaging effects of drought. It was onserved that 370 unigenes can encode LEA. We observed 19 DEGs related to LEAs in Axonopus compressus all of which were up-regulated under stress. Therefore, the unigenes are crucial for developing sustainability against the water shortage for Axonopus compressus. Additionally, the osmotic regulation ability of plants to hamper water loss is an additional fundamental feature to survive in drought. The bidirectional water channel AQP (aquaporin) in plants is responsible for trans-membrane water mobility and long-distance water transportation. It is established that plants have the potential to defy the numerous stress circumstances through regulating the AQP proteins [58]. In Axonopus compressus, we observed 363 
unigenes coding AQPs, including 31 differentially expressed unigenes. Majority of AQP's showed a reduced expression, which indicates a potential decline in AQP's activity. The decrease in AQPs expression is expected to correlate with limited moisture loss. It may sustain water-related homeostatic processes. Consequently, improving tolerance against drought in Axonopus compressus.

\section{Conclusion}

Under drought conditions, processing of stress signals, signal transduction, regulating gene expression and the subsequent downstream functional genes are essential variables in plant response to adversity. In current study, we observed 263,835 unigenes in Axonopus compressus based on RNA-Seq, in which 7445 were differentially expressed unigenes (DS and CK). Overall, 2747 were up-regulated and 2502 down-regulated unigenes. In addition, 352 (TFs) were found to be differentially expressed in Axonopus compressus (DS and CK). Current findings indicate that these genes are involved in the campaign of resistance to drought stress and have a highly significant and complicated role. This study provides valuable data for the molecular mechanisms underlying drought tolerance. The qRT-PCR validation of transcripts sets the basis for further investigations of the gene regulatory networks under drought stress and other abiotic stress factors in Axonopus compressus.

\section{Supplementary Information}

The online version contains supplementary material available at https://doi. org/10.1186/s12870-021-02844-7.

\section{Additional file 1.}

Additional file 2.

Additional file 3.

\section{Abbreviations}

DEGs: Differentially expressed genes; DEPs: Differentially expressed proteins; FPKM: Fragments Per Kilobase Million; GO: Gene Ontology; KO: KEGG Orthology; GST: Glutathione S-transferase; DS: Drought Stress; CK: Control; NGS: Next Generation Sequencing; KEGG: Kyoto Encyclopedia of Genes and Genomes; MAPK: Mitogen-activated protein kinase; NDEGs: No significant difference in genes.; RNA-seq: RNA sequencing.; ROS: Reactive oxygen species.; MDA: Malondialdehyde.; EL: Electrolyte Leakage.; CDNA: Complementary DNARNA-SeqRNA sequencingDEGsDifferentially expressed genes.; CDSs: CoDing Sequences.; ORF: Open Reading FramencRNAsNon-coding RNAs.; DE: Differentially expressedTFsTranscription factors.; BP: Biological processes.; CC: Cellular components.; MF: Molecular functions.; POD: Peroxidase.; SOD: Superoxide Dismutase.; CAT: Catalase.; LEA: Later Embryogenesis Abundant:; ABA: Abscisic acid.; PYR/PYL/ RCAR: Pyrabactin Resistance1/Pyr1-Like/Regulatory Components of Aba Receptors.; PP2C: Protein Phosphatase type 2C.; SnRK2: Serine/Threonine Kinases2.; SNF1: Sucrose Non-fermentation 1.; PEG: Polyethylene glycol.; LWP: Leaf water potential.; dNTP: Deoxynucleoside triphosphate.; qRTPCR: Real-Time Quantitative Reverse Transcription PCR.

\section{Acknowledgements}

The authors are grateful to the editors and referees for their valuable comments to improve our manuscript.

\section{Authors' contributions}

Conceptualization: MN, ZW, Data curation: MN, Formal analysis: MN, FA, AZ, Funding acquisition: MN, ZW, Investigation: MN, LL, Methodology: MN, Writing - original draft: MN, FA, YH, LL, Writing - review \& editing: MN, FA, ZW, SS, UA. All authors have read and approved the manuscript.

\section{Funding}

This work was supported by the Construction of World First Class Discipline of Hainan University (No.RZZX201905), the National Natural Science Foundation of China (31060266), the Hainan Provincial Natural Science Foundation (310029). National Project on Sci-Tec Foundation Resources Survey (2017FY100600). The authors are grateful to the editors and referees for their valuable comments to improve their manuscript.

\section{Availability of data and materials}

The datasets generated and/or analysed during the current study are included in this article, its supplementary information files and in the [NCBI] repository with Accession: PRJNA688376 [https://www.ncbi.nlm.nih.gov/sra/ PRJNA688376].

Ethics approval and consent to participate

Not applicable.

\section{Consent for publication}

Not applicable.

\section{Competing interests}

The authors declare that they have no competing interests.

\section{Author details}

'Key Laboratory of Genetics and Germplasm Innovation of Tropical Special Forest Trees and Ornamental Plants, Ministry of Education, College of Forestry and College of Tropical Crops, Hainan University, Haikou 570228, People's Republic of China. ${ }^{2}$ Department of Bioinformatics and Biotechnology, Govt. College University, Faisalabad, Pakistan. ${ }^{3}$ Key Laboratory of Development and Application of Rural Renewable Energy, Biomass Energy Technology Research Centre, Biogas Institute of Ministry of Agriculture, South Renmin Road, Chengdu 610041, China. ${ }^{4}$ Adaptive Research Farm, Gujranwala 52250, Pakistan. ${ }^{5}$ Department of Botany, University of Education, Lahore, Faisalabad-Campus, Faisalabad 38000, Pakistan. ${ }^{6}$ Tropical Crops Genetic Resources Institute, Chinese Academy of Tropical Agricultural Sciences, Danzhou 571737, Hainan, China.

Received: 14 July 2020 Accepted: 19 January 2021

Published online: 02 February 2021

\section{References}

1. López A, Morrone O. Phylogenetic studies in Axonopus (Poaceae, Panicoideae, Paniceae) and related genera: morphology and molecular (nuclear and plastid) combined analyses. Syst Bot. 2012;37:671-6.

2. Ighovie ES, Ikechukwu EE. Phytoremediation of crude oil contaminated soil with Axonopus compressus in the Niger Delta region of Nigeria. Nat Resour. 2014;5:59-67. https://doi.org/10.4236/nr.2014.52006.

3. Wang $Z Y, H u H G$, Bai CJ, Wang XL, Mo DQ, Li LF, et al. Genetic variation of asexual reproduction characteristics of Axonopus compressus (Sw.) P. Beauv. In: VI International Symposium on the Taxonomy of Cultivated Plants 1035. 2013. p. 189-203.

4. Hewins DB, Lyseng MP, Schoderbek DF, Alexander M, Willms WD, Carlyle $\mathrm{CN}$, et al. Grazing and climate effects on soil organic carbon concentration and particle-size association in northern grasslands. Sci Rep. 2018;8:1336.

5. Knapp AK, Carroll CJW, Denton EM, La Pierre K, Collins SL, Smith MD. Differential sensitivity to regional-scale drought in six central US grasslands. Oecologia. 2015;177:949-57.

6. Farooq M, Wahid A, Kobayashi N, Fujita D, Basra SMA. Plant drought stress: effects, mechanisms and management. In: Sustainable Agriculture. Dordrecht: Springer Netherlands; 2009. p. 153-88. https://doi.org/10.1007/ 978-90-481-2666-8_12.

7. Deng X, Liu K, Li M, Zhang W, Zhao X, Zhao Z, et al. Difference of selenium uptake and distribution in the plant and selenium form in the grains of rice with foliar spray of selenite or selenate at different stages. F Crop Res. 2017; 211:165-71 
8. Lei T, Pang Z, Wang X, Li L, Fu J, Kan G, et al. Drought and carbon cycling of grassland ecosystems under global change: a review. Water. 2016;8:460

9. Wang XL, Li Y, Liao L, Bai CJ, Wang ZY. Isolation and characterization of microsatellite markers for Axonopus compressus (Sw.) Beauv.(Poaceae) using 454 sequencing technology. Genet Mol Res. 2015;14:4696-702.

10. Porse A, Schou TS, Munck C, Ellabaan MMH, Sommer MOA. Biochemical mechanisms determine the functional compatibility of heterologous genes. Nat Commun. 2018;9:522.

11. Byeon Y, Lee H, Lee HY, Back K. Cloning and functional characterization of the Arabidopsis $\mathrm{N}$-acetylserotonin O-methyltransferase responsible for melatonin synthesis. J Pineal Res. 2016;60:65-73.

12. Gierahn TM, Wadsworth MH II, Hughes TK, Bryson BD, Butler A, Satija R, et al. Seq-well: portable, low-cost RNA sequencing of single cells at high throughput. Nat Methods. 2017;14:395.

13. Adams IP, Fox A, Boonham N, Massart S, De Jonghe K. The impact of high throughput sequencing on plant health diagnostics. Eur J Plant Pathol. 2018;152:909-19.

14. Tang X, Liu G, Zhou J, Ren Q, You Q, Tian L, et al. A large-scale wholegenome sequencing analysis reveals highly specific genome editing by both Cas9 and Cpf1 (Cas12a) nucleases in rice. Genome Biol. 2018;19:84.

15. Srivastava D, Verma G, Chauhan AS, Pande V, Chakrabarty D. Rice (Oryza sativa L.) tau class glutathione S-transferase (OsGSTU30) overexpression in Arabidopsis thaliana modulates a regulatory network leading to heavy metal and drought stress tolerance. Metallomics. 2019;11:375-89.

16. Dubois $M$, Claeys $H$, Van den Broeck L, Inzé $D$. Time of day determines Arabidopsis transcriptome and growth dynamics under mild drought. Plant Cell Environ. 2017:40:180-9.

17. Zotova L, Kurishbayev A, Jatayev S, Khassanova G, Zhubatkanov A, Serikbay $D$, et al. Genes encoding transcription factors TaDREB5 and TaNFYC-A7 are differentially expressed in leaves of bread wheat in response to drought dehydration and ABA. Front Plant Sci. 2018;9:1441.

18. Janiak A, Kwasniewski M, Sowa M, Kuczyńska A, Mikołajczak K, Ogrodowicz $P$, et al. Insights into barley root Transcriptome under mild drought stress with an emphasis on gene expression regulatory mechanisms. Int J Mol Sci. 2019;20:6139.

19. Liu X, Zhang X, Sun B, Hao L, Liu C, Zhang D, et al. Genome-wide identification and comparative analysis of drought-related microRNAs in two maize inbred lines with contrasting drought tolerance by deep sequencing. PLoS One. 2019;14.

20. Gosa SC, Lupo Y, Moshelion M. Quantitative and comparative analysis of whole-plant performance for functional physiological traits phenotyping: new tools to support pre-breeding and plant stress physiology studies. Plant Sci. 2019;282:49-59

21. Min $H$, Chen $C$, Wei $S$, Shang $X$, Sun $M$, Xia R, et al. Identification of drought tolerant mechanisms in maize seedlings based on transcriptome analysis of recombination inbred lines. Front Plant Sci. 2016;7:1080.

22. Harris-Shultz KR, Hayes CM, Knoll JE. Mapping QTLs and identification of genes associated with drought resistance in Sorghum. In: Sorghum. Springer; 2019. p. 11-40.

23. Scharwies JD, Dinneny JR. Water transport, perception, and response in plants. J Plant Res. 2019;132:311-24.

24. Li J, Yu G, Sun X, Liu Y, Liu J, Zhang X, et al. AcPIP2, a plasma membrane intrinsic protein from halophyte Atriplex canescens, enhances plant growth rate and abiotic stress tolerance when overexpressed in Arabidopsis thaliana. Plant Cell Rep. 2015;34:1401-15.

25. Fei X, Hou L, Shi J, Yang T, Liu Y, Wei A. Patterns of drought response of 38 WRKY transcription factors of Zanthoxylum bungeanum maxim. Int J Mol Sci. 2019;20:68.

26. Nawaz M, Wang Z. Abscisic acid and Glycine Betaine mediated tolerance mechanisms under drought stress and recovery in Axonopus compressus: a new insight. Sci Rep. 2020;10:1-10.

27. Liu N, Lin S, Huang B. Differential effects of Glycine Betaine and Spermidine on osmotic adjustment and antioxidant defense contributing to improved drought tolerance in creeping Bentgrass. J Am Soc Hortic Sci. 2017;142:20-6.

28. Simão FA, Waterhouse RM, loannidis P, Kriventseva EV, Zdobnov EM. BUSCO: assessing genome assembly and annotation completeness with single-copy orthologs. Bioinformatics. 2015;31:3210-2.

29. Spokevicius AV, Tibbits J, Rigault P, Nolin M-A, Müller C, Merchant A. Medium term water deficit elicits distinct transcriptome responses in Eucalyptus species of contrasting environmental origin. BMC Genomics. 2017;18:284
30. Saddhe AA, Malvankar MR, Karle SB, Kumar K. Reactive nitrogen species: paradigms of cellular signaling and regulation of salt stress in plants. Environ Exp Bot. 2019;161:86-97.

31. Hasanuzzaman M, Shabala L, Brodribb TJ, Zhou M, Shabala S. Understanding physiological and morphological traits contributing to drought tolerance in barley. J Agron Crop Sci. 2019;205:129-40.

32. Anjum SA, Ashraf U, Tanveer M, Khan I, Hussain S, Shahzad B, et al. Drought induced changes in growth, Osmolyte accumulation and antioxidant metabolism of three maize hybrids. Front Plant Sci. 2017;08. https://doi.org/ 10.3389/fpls.2017.00069.

33. Jiang Y, Huang B. Protein Alterations in Tall Fescue in Response to Drought Stress and Abscisic Acid. Crop Sci. 2002;42:202-7. doi:doi:https://doi.org/10. 2135/cropsci2002.2020.

34. Gujjar RS, Akhtar M, Rai A, Singh M. Expression analysis of droughtinduced genes in wild tomato line (Solanum habrochaites). Curr Sci. 2014;107:496-501.

35. Cantalapiedra CP, García-Pereira MJ, Gracia MP, Igartua E, Casas AM, Contreras-Moreira B. Large Differences in Gene Expression Responses to Drought and Heat Stress between Elite Barley Cultivar Scarlett and a Spanish Landrace. Front Plant Sci. 2017;8 May.

36. Osakabe Y, Osakabe K, Shinozaki K, Tran L-SP. Response of plants to water stress. Front Plant Sci. 2014:5:86.

37. Yadav A, Stobdan T, Chauhan OP, Dwivedi SK, Chaurasia OP. Sea buckthorn: a multipurpose medicinal plant from upper Himalayas. In: Medicinal Plants. Springer; 2019. p. 399-426.

38. Ding F, Wang G, Wang M, Zhang $S$. Exogenous melatonin improves tolerance to water deficit by promoting cuticle formation in tomato plants. Molecules. 2018;23:1605.

39. Bi H, Kovalchuk N, Langridge P, Tricker PJ, Lopato S, Borisjuk N. The impact of drought on wheat leaf cuticle properties. BMC Plant Biol. 2017;17:85.

40. Ziv C, Zhao Z, Gao YG, Xia Y. Multifunctional roles of plant cuticle during plant-pathogen interactions. Front Plant Sci. 2018;9:1088.

41. Nguyen D, Rieu I, Mariani C, van Dam NM. How plants handle multiple stresses: hormonal interactions underlying responses to abiotic stress and insect herbivory. Plant Mol Biol. 2016;91:727-40.

42. Tombesi S, Nardini A, Frioni T, Soccolini M, Zadra C, Farinelli D, et al. Stomatal closure is induced by hydraulic signals and maintained by ABA in drought-stressed grapevine. Sci Rep. 2015;5:1-12.

43. Martorell S, DIAZ-ESPEJO A, Medrano H, Ball MC, Choat B. Rapid hydraulic recovery in $\mathrm{E}$ ucalyptus pauciflora after drought: linkages between stem hydraulics and leaf gas exchange. Plant Cell Environ. 2014;37:617-26.

44. Dubois $\mathrm{M}$, Inzé D. Plant growth under suboptimal water conditions: early responses and methods to study them. J Exp Bot. 2020.

45. Dittrich M, Mueller HM, Bauer H, Peirats-Llobet M, Rodriguez PL, Geilfus C-M, et al. The role of Arabidopsis ABA receptors from the PYR/PYL/RCAR family in stomatal acclimation and closure signal integration. Nat plants. 2019;5: 1002-11.

46. Yu J, Yang L, Liu X, Tang R, Wang Y, Ge H, et al. Overexpression of poplar pyrabactin resistance-like abscisic acid receptors promotes abscisic acid sensitivity and drought resistance in transgenic Arabidopsis. PLoS One. 2016;11.

47. Ye G, Ma Y, Feng Z, Zhang X. Transcriptomic analysis of drought stress responses of sea buckthorn (Hippophae rhamnoidessubsp. Sinensis) by RNA-Seq. PLoS One. 2018;13.

48. Sah SK, Reddy KR, Li J. Abscisic acid and abiotic stress tolerance in crop plants. Front Plant Sci. 2016;7:571.

49. Liu J-H, Peng T, Dai W. Critical cis-acting elements and interacting transcription factors: key players associated with abiotic stress responses in plants. Plant Mol Biol Report. 2014;32:303-17.

50. Qureshi MK, Munir S, Shahzad AN, Rasul S, Nouman W, Aslam K. Role of reactive oxygen species and contribution of new players in defense mechanism under drought stress in rice. Int J Agric Biol. 2018:20:1339-52

51. Rokhzadi A. Response of chickpea (Cicer arietinum L.) to exogenous salicylic acid and ascorbic acid under vegetative and reproductive drought stress conditions. J Appl bot. Food Qual. 2014;87.

52. Gahlaut V, Jaiswal V, Kumar A, Gupta PK. Transcription factors involved in drought tolerance and their possible role in developing drought tolerant cultivars with emphasis on wheat (Triticum aestivum L.). Theor Appl Genet. 2016;129:2019-42

53. Kulkarni M, Soolanayakanahally R, Ogawa S, Uga Y, Selvaraj MG, Kagale S. Drought response in wheat: key genes and regulatory mechanisms 
controlling root system architecture and transpiration efficiency. Front Chem. 2017;5:106.

54. Samad AFA, Sajad M, Nazaruddin N, Fauzi IA, Murad A, Zainal Z, et al. MicroRNA and transcription factor: key players in plant regulatory network. Front Plant Sci. 2017;8:565.

55. Sun H, Xia B, Wang X, Gao F, Zhou Y. Quantitative phosphoproteomic analysis provides insight into the response to short-term drought stress in Ammopiptanthus mongolicus roots. Int J Mol Sci. 2017;18:2158.

56. Bhaskara GB, Nguyen $T$, Yang $T-H$, Verslues PE. Comparative analysis of phosphoproteome remodeling after short term water stress and ABA treatments versus longer term water stress acclimation. Front Plant Sci. 2017;8:523.

57. Ma H, Chen J, Zhang Z, Ma L, Yang Z, Zhang Q, et al. MAPK kinase 10.2 promotes disease resistance and drought tolerance by activating different MAPKs in rice. Plant J. 2017:92:557-70.

58. Quiroga G, Erice G, Aroca R, Zamarreño ÁM, García-Mina JM, Ruiz-Lozano JM. Radial water transport in arbuscular mycorrhizal maize plants under drought stress conditions is affected by indole-acetic acid (IAA) application. J Plant Physiol. 2020;:153115.

\section{Publisher's Note}

Springer Nature remains neutral with regard to jurisdictional claims in published maps and institutional affiliations.

Ready to submit your research? Choose BMC and benefit from:

- fast, convenient online submission

- thorough peer review by experienced researchers in your field

- rapid publication on acceptance

- support for research data, including large and complex data types

- gold Open Access which fosters wider collaboration and increased citations

- maximum visibility for your research: over $100 \mathrm{M}$ website views per year

At $\mathrm{BMC}$, research is always in progress.

Learn more biomedcentral.com/submissions 